\title{
Managing refractory cryoglobulinemic vasculitis: challenges and solutions
}

This article was published in the following Dove Press journal:

Journal of Inflammation Research

8 May 2017

Number of times this article has been viewed

\section{Predrag Ostojic \\ Ivan $\mathrm{R}$ Jeremic}

Institute of Rheumatology, School of Medicine, University of Belgrade, Belgrade, Serbia
Correspondence: Predrag Ostojic Institute of Rheumatology, School of Medicine, University of Belgrade, Resavska 69, I 1000 Belgrade, Serbia Email drpedja74@hotmail.com
Abstract: Cryoglobulinemia is thought to be a rare condition. It may be an isolated disorder or secondary to a particular disease. According to immunoglobulin composition, cryoglobulinemia is classified into three types. In mixed cryoglobulinemia (types II and III), vascular deposition of cryoglobulin-containing immune complexes and complement may induce a clinical syndrome, characterized by systemic vasculitis and inflammation - cryoglobulinemic vasculitis (CryoVas). Most common clinical manifestations in CryoVas are skin lesions (orthostatic purpura and ulcers), weakness, peripheral neuropathy, Raynaud's phenomenon, sicca syndrome, membranoproliferative glomerulonephritis, and arthralgia and seldom arthritis. In patients with mixed cryoglobulinemia, prevalence of anti-hepatitis $\mathrm{C}$ virus (HCV) antibodies and/or HCV RNA, detected by polymerase chain reaction (PCR), is reported to be up to $90 \%$, indicating a significant role of $\mathrm{HCV}$ in the development of this condition. The goals of therapy for mixed cryoglobulinemia include immunoglobulin level reduction and antigen elimination. CryoVas not associated with $\mathrm{HCV}$ infection should be treated according to treatment recommendations for small-vessel vasculitides. CryoVas associated with chronic HCV infection should be treated with antivirals along with immunosuppressive drugs, with or without plasmapheresis, depending on disease severity and organ involvement. Patients who do not respond to first-line therapy may achieve remission when treatment with rituximab is started as second-line therapy. In HCVrelated CryoVas, antiviral therapy should be given along with rituximab in order to achieve complete or partial remission. Moreover, rituximab has proven to be a glucocorticoid-sparing medication. Other potential therapies for refractory CryoVas include mycophenolate mofetil and belimumab, while tumor necrosis factor (TNF) inhibitors are not effective.

Keywords: cryoglobulinemia, vasculitis, treatment

\section{Introduction}

Cryoglobulins are proteins that become insoluble and typically precipitate at temperatures below normal body temperature and dissolve back into the serum upon rewarming. Cryoglobulins are either immunoglobulins or a mixture of immunoglobulins and complement components. ${ }^{1}$ They should be distinguished from cryofibrinogen, which is typically composed of a mixture of fibrinogen, fibrin, fibronectin, and fibrin split products. When patient's serum and plasma, refrigerated at $4^{\circ} \mathrm{C}$ for up to 72 hours, form a precipitate, then the precipitated proteins are referred to as cryoglobulins. On the other hand, cryofibrinogen precipitates only in plasma and not in the serum. Cryofibrinogenemia may be primary (essential) or secondary to other underlying disorders, such as carcinoma, infection, vasculitis, and connective tissue disease, or associated with cryoglobulinemia. ${ }^{2}$ 


\section{Classification of cryoglobulinemia}

Cryoglobulinemia can be associated with various diseases such as multiple myeloma, Waldenström's macroglobulinemia, lymphoma, hepatitis $\mathrm{C}$ virus (HCV) infection, and connective tissue diseases (primarily rheumatoid arthritis and Sjögren's syndrome). Cryoglobulinemia associated with a particular disease is known as secondary cryoglobulinemia. On the other hand, it may be an isolated condition, named essential (or idiopathic) cryoglobulinemia.

According to immunoglobulin composition, cryoglobulinemia is classified into three types: type I cryoglobulinemia (composed of only one monoclonal immunoglobulin, usually immunoglobulin $\mathrm{M}$ [IgM] or, less frequently, immunoglobulin $\mathrm{G}$ [IgG], immunoglobulin A [IgA], or light chains) and type II and type III cryoglobulinemia (commonly termed as mixed cryoglobulinemia, containing IgM rheumatoid factor [RF], an antibody with activity toward the Fc fragment of IgG immunoglobulins). ${ }^{1}$ Type II cryoglobulinemia is characterized by monoclonal immunoglobulins with $\mathrm{RF}$ activity, while polyclonal RF is typically present in type III cryoglobulinemia. Type I cryoglobulinemia is associated with lymphoma, Waldenström's macroglobulinemia, and multiple myeloma. On the other hand, mixed cryoglobulinemia may be associated with infections (mainly hepatitis C) and autoimmune or lymphoproliferative disorders and rarely may be idiopathic (essential). Chronic HCV infection was found to be the underlying disorder in most of the cases of mixed cryoglobulinemia. The prevalence of serum anti-HCV antibodies and/or HCV RNA, detected by polymerase chain reaction (PCR), in patients with mixed cryoglobulinemia is reported to be between $70 \%$ and $90 \%$, indicating a leading role of $\mathrm{HCV}$ in the development of this rare condition. ${ }^{3,4} \mathrm{HCV}$ probably exerts a chronic stimulus of the immune system and may trigger RF production. Moreover, HCV may be involved in the pathogenesis of organ-specific and systemic autoimmune diseases, as well as lymphoproliferative disorders. It was shown that antiviral therapy improves the outcomes of HCV-associated B-cell non-Hodgkin lymphoma (NHL), which confirms the causal relationship between HCV infection and the development of NHL. ${ }^{5}$ Therefore, all patients with cryoglobulinemia, regardless of the underlying disorder, should be tested for HCV infection.

\section{Pathogenetic role of cryoglobulins}

Cryoglobulins can cause organ and tissue damage by several mechanisms. Circulating large cryoproteins may lead to increased blood viscosity, which may result in hypoperfusion. This mechanism is commonly seen in type
I cryoglobulinemia, primarily in Waldenström's macroglobulinemia and multiple myeloma. ${ }^{6}$ Confusion, mental status changes, and neurologic symptoms result from decreased cerebral blood flow. Other clinical manifestations include mucosal bleeding, visual changes, and cardiopulmonary symptoms (shortness of breath, hypoxemia, and congestive heart failure). However, patients with type I cryoglobulinemia are asymptomatic until the level of cryoglobulins is sufficiently high to cause hyperviscosity syndrome.

Another mechanism of tissue damage is cryoprecipitation in vivo, with plugging and thrombosis of small arteries and capillaries in the extremities (causing gangrene) and glomeruli (leading to acute renal failure).

In mixed cryoglobulinemia (types II and III), vascular deposition of cryoglobulin-containing immune complexes and complement may induce a clinical syndrome, characterized by systemic vasculitis and inflammation - cryoglobulinemic vasculitis (CryoVas). ${ }^{7}$ CryoVas may be classified in the subgroup of small-vessel systemic vasculitides. The leukocytoclastic vasculitis is the histopathological hallmark of CryoVas. ${ }^{8}$ While complement activation by immune complexes is probably the main pathogenic pathway of CryoVas, contribution of hemorheological disturbances due to high blood viscosity and cryoprecipitation should not be underestimated in mixed cryoglobulinemia. ${ }^{9}$ Furthermore, it was shown that vasculitis in cryoglobulinemia can be caused by immunoglobulins in the absence of antigens, indicating the possibility of a pathogenic pathway distinct from immune complex-mediated inflammation. ${ }^{10}$ Chronic $\mathrm{HCV}$ infection is the most frequent cause of mixed cryoglobulinemia and CryoVas.

\section{Clinical features of CryoVas}

Cryoglobulinemia is thought to be a rare condition. The prevalence of essential mixed cryoglobulinemia is reported to be $\sim 1: 100,000$, with a female-to-male ratio of $3: 1{ }^{11}$ Mixed cryoglobulinemia (types II and III) represent nearly $75 \%$ of all cases. ${ }^{1}$ Symptoms and signs of CryoVas vary among patients. Most common clinical manifestations, present in $>90 \%$ of patients, include skin lesions, in particular orthostatic purpura and ulcers, weakness, and arthralgia., ${ }^{4}$ Peripheral neuropathy and chronic hepatitis due to $\mathrm{HCV}$ infection (which is the most common underlying disorder in mixed cryoglobulinemia) are frequently found. ${ }^{13,14}$ Raynaud's phenomenon and Sicca syndrome are present in 30-50\% of patients, while non-erosive arthritis is rare. ${ }^{12}$ However, prognosis and survival mainly depend on kidney involvement (membranoproliferative glomerulonephritis). Clinical 
manifestations of CryoVas are comparable in patients with type II and type III mixed cryoglobulinemia. ${ }^{4}$ Prognosis also depends on the underlying disease. For instance, patients with chronic $\mathrm{HCV}$ infection have a better prognosis if they respond to antiviral therapy. On the other hand, patients with renal disease or lymphoproliferative or plasma cell disorders have a worse prognosis.

The detection of mixed (IgG-IgM) cryoglobulins in the serum is necessary for a correct diagnosis of mixed cryoglobulinemia. A combination of circulating mixed cryoglobulins, low $\mathrm{C} 4$, and orthostatic skin purpura is highly suggestive of CryoVas. Whenever possible, skin biopsy of recent vasculitic lesions should be done. A leukocytoclastic vasculitis, involving small-sized blood vessels (arterioles, capillaries, and venules), is the typical pathological finding. ${ }^{4}$

\section{Treatment goals for CryoVas}

Treatment strategy in patients with cryoglobulinemia depends on the type of the disease. In secondary cryoglobulinemia, treatment of the underlying disease is crucial. For instance, chemotherapy usually reduces immunoglobulin levels and their contribution to hyperviscosity syndrome in patients with lymphoproliferative disorders and type I cryoglobulinemia. On the other hand, in mixed cryoglobulinemia (type II and type III), treatment strategy is mainly based on anti-inflammatory and immunosuppressive therapies. The goals of therapy for CryoVas include immunoglobulin level reduction and antigen elimination. The first objective may be achieved using immunosuppressive medications, while the second objective depends on whether the antigen is known or not. In HCV-associated CryoVas, antiviral therapy reduces antigens. However, in autoimmune diseases, a potential antigen is usually unrecognized and nonspecific immunosuppressive drugs are only used.

\section{Treatment of CryoVas associated with HCV infection}

CryoVas associated with chronic HCV infection should be treated with antivirals along with immunosuppressive drugs, with or without plasmapheresis, depending on disease severity and organ involvement. ${ }^{15}$ Interferon-free treatment with direct-acting antiviral drugs is recommended for most $\mathrm{HCV}$ genotypes. A combination of interferon-free antiviral drugs (sofosbuvir plus ribavirin) improves skin, peripheral nerve, and joint and kidney manifestations in HCV-related CryoVas. ${ }^{16}$ However, treatment for patients with HCV genotype 6 involves direct-acting antiviral drugs, taken with pegylated interferon injections. First-generation protease inhibitors are no longer recommended. ${ }^{17}$
The use of immunosuppressive drugs in the treatment of CryoVas, associated with chronic HCV infection, may worsen the underlying infection. However, antiviral agents as the only therapy are not sufficient to treat clinical manifestations of vasculitis. Reaching just one of the treatment goals - reduction of antigens - by using anti-infective drugs only may result in less remission and higher possibility of relapses. Moreover, despite the successful elimination of viral antigens, vasculitis can persist, as secreted immunoglobulins may have properties of cryoglobulins in the absence of complementary antigens. ${ }^{10}$ It is not completely clarified, whether antiviral and immunosuppressive treatments should be administered simultaneously or sequentially, due to possible adverse events. Some authors suggest only antiviral drugs in mild forms of HCV-related CryoVas, while in patients with severe vasculitis rituximab, with or without plasmapheresis, should be used in combination with antiviral therapy. Other immunosuppressive agents are recommended only in cases of refractory CryoVas. ${ }^{18}$ In patients with membranoproliferative glomerulonephritis, as a clinical feature of HCV-related CryoVas, immunosuppressive drugs are recommended only in case of nephrotic syndrome or rapidly progressive renal failure. In milder forms of nephropathy, only antivirals should be used. ${ }^{19,20} \mathrm{We}$ are still searching for an ideal effective and safe combination of antiviral and immunosuppressive drugs, since CryoVas is a rare disease, with only few controlled clinical trials in the literature. This applies in particular to CryoVas associated with autoimmune diseases.

\section{Treatment of CryoVas not associated with HCV infection}

CryoVas not associated with HCV infection should be treated according to treatment recommendations for small-vessel vasculitides. Therapy depends on severity of initial manifestations. In life-threatening diseases, immunosuppression with glucocorticoids and cyclophosphamide pulse therapy, with or without plasmapheresis, is advised as initial therapy. In mild disease manifestations, methotrexate as a less toxic immunosuppressive drug is recommended. ${ }^{15}$

\section{Assessment of treatment response}

Evaluation of treatment response is difficult because published studies are heterogeneous, in terms of inclusion criteria (patients have different clinical manifestations of CryoVas at baseline), remission criteria (clinical, viral, and immunological), and immunosuppressive drugs that were given. ${ }^{21-23}$ To define remission, in some studies, the Birmingham Vasculitis 
Activity Score (BVAS) was used as an assessment tool. ${ }^{21,22,24}$ In other studies, efficacy of treatment was estimated by evaluation of particular clinical manifestation - orthostatic purpura and/or skin ulcers, peripheral neuropathy, arthralgia, serum creatinine, proteinuria, and/or hematuria in patients with renal involvement. ${ }^{24-28}$ Virological remission was evaluated by levels of HCV RNA in serum, as well as in the cryoprecipitate..$^{21,24-28}$ Some studies measured the level of depletion of B-lymphocytes after administration of rituximab. ${ }^{21,25,26,28}$ Some authors assessed the levels of cryoglobulins and RF as immunological parameters of treatment efficacy. ${ }^{21,24-26,28}$ Interestingly, one study did not find any relationship between levels of cryoglobulins and vasculitis relapse. ${ }^{21}$

\section{Rituximab in CryoVas associated with HCV infection}

Rituximab is a monoclonal antibody that binds to the CD20 protein on the surface of B-cells in various stages of differentiation. Mature and activated plasma cells do not contain CD20 on their surface and therefore are not targeted by the drug. The positive effect on CryoVas is achieved by depletion of B lymphocytes, which produce polyclonal or monoclonal RF in type II and type III cryoglobulinemia. Consequently, rituximab reduces formation of immune complexes. In two studies, patients with $\mathrm{HCV}$-associated CryoVas, affecting the skin, peripheral nerves, and kidneys, who did not respond or did not tolerate therapy with interferon-alpha and ribavirin, were treated with rituximab. ${ }^{21,22}$ In the first study, rituximab was applied at a dose of $375 \mathrm{mg} / \mathrm{m}^{2}$ weekly for 4 weeks. ${ }^{21}$ Efficacy and safety of rituximab, added to previous ineffective immunosuppressive therapy, were compared with results of treatment in a control group. Patients in the control group were maintained on any immunosuppressive therapies that they were receiving at the time of enrollment and were allowed to increase or initiate new immunosuppressive treatments as needed to manage worsening disease activity. After 6 months of follow-up, remission was achieved in $83 \%$ of patients in the rituximab subgroup compared to only $8 \%$ of patients treated with other conventional immunosuppressive drugs. ${ }^{21}$ In the second study, patients were treated with two doses of $1,000 \mathrm{mg}$, separated by 2 weeks. ${ }^{22}$ Drug survival after 24 months was significantly higher in patients treated with rituximab compared to those treated with other immunosuppressive medications (64\% vs 3.5\%). ${ }^{22}$ In both studies, rituximab has proven to be successful as a drug with glucocorticoid-sparing effect. In addition, if used after conventional immunosuppressive treatment, rituximab was less effective to induce remission, with more frequent relapses, compared to patients treated with rituximab as an initial immunosuppressive drug. ${ }^{22}$ In both studies, rituximab did not cause worsening of the underlying infectious disease..$^{21,22}$

Some trials assessed efficacy of the PIRR protocol (concomitant treatment with Pegylated Interferon, Ribavirin, and Rituximab) in patients with HCV-associated CryoVas. The PIRR protocol showed to be more effective as an initial treatment in patients with skin, peripheral nerve, and kidney involvement compared to antiviral therapy with pegylated interferon and ribavirin only. ${ }^{28}$ Complete remission was noticed more frequently among patients treated with PIRR (54.5\% vs $33.3 \%$ ). Cryoglobulinemia was more evident in patients treated with antivirals only, despite the HCV antigen reduction. Moreover, long-lasting positive effects of the PIRR protocol have been proven. ${ }^{28}$ In another study, PIRR protocol was compared to rituximab monotherapy. Compared to patients on rituximab monotherapy, more patients treated according to the PIRR protocol achieved complete clinical remission ( $80 \%$ vs 58\%), partial clinical remission ( $15 \%$ vs $9 \%$ ), and complete immunological remission $(67 \%$ vs $46 \%$ ). Virological remission was achieved in $55 \%$ of patients treated with PIRR protocol. ${ }^{25}$ Several case reports presented successful use of rituximab in severe and refractory forms of CryoVas associated with $\mathrm{HCV}$ infection, to treat different clinical manifestations or to treat patients with significant comorbidities such as compensated liver cirrhosis or lymphoma. ${ }^{29-35}$

\section{Rituximab in CryoVas not associated with HCV infection}

Patients with CryoVas not related to HCV infection, affecting skin, peripheral nervous system, and kidneys (two-third with severe renal involvement), refractory to first-line therapy with glucocorticosteroids and azathioprine, were afterward successfully treated with rituximab and retreated when neccessary. ${ }^{36}$ In another retrospective study, rituximab proved to be effective in the treatment of nephropathy caused by CryoVas (improvement in $95 \%$ of patients, complete remission in $50 \%$ of patients) and in one patient with abdominal vasculitis, which is a severe form of CryoVas. ${ }^{37}$ Data from the CryoVas survey demonstrated a significant corticosteroidsparing effect of rituximab in patients with this form of the disease. ${ }^{38}$ Efficacy in non-HCV infection-associated CryoVas was also demonstrated in case reports. ${ }^{36,39}$ One case report showed efficacy of rituximab in CryoVas associated with hepatitis B viral infection, which is a quite rare condition. ${ }^{40}$ 


\section{Safety of rituximab in CryoVas}

According to several studies, rituximab seems to be a safe medication in patients with CryoVas, including in those with chronic HCV infection..$^{29,31,34-36,40}$ Severe adverse effects (fulminant hepatitis in HCV liver cirrhosis, serious infections, and sepsis) were rarely noticed when applied for primary glomerulonephritis treatment. ${ }^{26,38,41,42}$ Respiratory infections were slightly more frequent, as well as cytopenias, when rituximab was applied along with antiviral drugs. ${ }^{32,33,43}$ In some patients with HCV-related CryoVas, severe systemic reactions after administration of rituximab have been noticed. ${ }^{37,44}$ It is believed that rituximab may form a complex with IgM-kappa mixed cryoglobulin and induce systemic reactions. ${ }^{44}$

\section{Other potential therapies}

Successful use of mycophenolate mofetil was shown in a patient with severe CryoVas related to systemic sclerosis and secondary Sjögren's syndrome, refractory to previous treatment with glucocorticoids and cyclophosphamide ${ }^{45} \mathrm{Clinical}$ features included finger-tip ulcers and gangrene, arthritis, renal involvement, and myocardial ischemia.

Inhibitors of tumor necrosis factor alpha (TNF- $\alpha$ ) are not recommended for the treatment of CryoVas. Infliximab, a monoclonal antibody against TNF- $\alpha$, was used in a patient with refractory CryoVas related to chronic HCV infection, with skin, peripheral nerve, and kidney involvement. Infliximab showed favorable effect on neuropathy, but skin manifestations relapsed after 4 months of treatment onset. ${ }^{46}$ Two more cases of CryoVas were treated with infliximab. The condition of one patient with skin changes and peripheral neuropathy worsened after third infliximab injection. He developed central nervous system symptoms with fatal outcome. No improvement was noticed after beginning of treatment with infliximab in the second patient, who had orthostatic purpura and skin ulcers, peripheral neuropathy, and glomerulonephritis. ${ }^{47}$

Another potentially useful biologic agent in the treatment of CryoVas is belimumab, a monoclonal antibody against the B-lymphocyte stimulator (BLyS). It was shown that different types of B-cell activating factor (BAFF) may contribute to production of cryoglobulins in chronic $\mathrm{HCV}$ infection. ${ }^{48,49}$ However, there are no published cases on the use of belimumab in patients with CryoVas so far.

\section{Disclosure}

The authors report no conflicts of interest in this work.

\section{References}

1. Brouet JC, Clouvel JP, Danon F, et al. Biologic and clinical significance of cryoglobulins. Am J Med. 1974;57:775-788.

2. Michaud M, Pourrat J. Cryofibrinogenemia. J Clin Rheumatol. 2013;19(3):142-148.

3. Mascia MT, Ferrari D, Campioli D, et al. Non HCV-related mixed cryoglobulinemia. Dig Liver Dis. 2007;39(suppl 1):S61-S64.

4. Ferri C, Mascia MT. Cryoglobulinemic vasculitis: review. Curr Opin Rheumatol. 2006;18:54-63.

5. Michot JM, Canioni D, Driss H, et al; ANRS HC-13 Lympho-C Study Group. Antiviral therapy is associated with a better survival in patients with hepatitis $\mathrm{C}$ virus and B-cell non-Hodgkin lymphomas, ANRS HC-13 lympho-C study. Am J Hematol. 2015;90(3):197-203.

6. Adams BD, Baker R, Lopez JA, Spencer S. Myeloproliferative disorders and the hyperviscosity syndrome. Emerg Med Clin North Am. 2009;27(3):459-476.

7. Ferri C, Zignego AL, Pileri SA. Cryoglobulins (review). J Clin Pathol. 2002;55:4-13.

8. Lamprecht P, Gause A, Gross WL. Cryoglobulinemic vasculitis (review) Arthritis Rheum. 1999;42:2507-2516.

9. Ferri C, Mannini L, Bartoli V, et al. Blood viscosity and filtration abnormalities in mixed cryoglobulinemia patients. Clin Exp Rheumatol. 1990;8(3):271-281.

10. Fabrizi F. Hepatitis $C$ virus, cryoglobulinemia, and kidney: novel evidence. Scientifica (Cairo). 2012;2012:11. Article ID 128382.

11. Gorevic PD, Kassab HJ, Levo Y, et al. Mixed cryoglobulinemia: clinical aspects and long-term follow-up of 40 patients. Am J Med. 1980;69(2):287-308.

12. Ferri C, Sebastiani M, Giuggioli D, et al. Mixed cryoglobulinemia: demographic, clinical, and serological features, and survival in 231 patients. Sem Arthritis Rheum. 2004;33:355-374.

13. Ammendola A, Sampaolo S, Ambrosone L, et al. Peripheral neuropathy in hepatitis-related mixed cryoglobulinemia: electrophysiologic followup study. Muscle Nerve. 2005;31(3):382-385.

14. Ferri C, Ramos-Casals M, Zignego AL, et al; ISG-EHCV coauthors. International diagnostic guidelines for patients with HCV-related extrahepatic manifestations. A multidisciplinary expert statement. Autoimmun Rev. 2016;15(12):1145-1160.

15. Mukhtyar C, Guillevin L, Cid MC, et al; European Vasculitis Study Group. EULAR recommendations for the management of primary small and medium vessel vasculitis. Ann Rheum Dis. 2009;68(3):310-317.

16. Saadoun D, Thibault V, Si Ahmed SN, et al. Sofosbuvir plus ribavirin for hepatitis $\mathrm{C}$ virus-associated cryoglobulinaemia vasculitis: VASCUVALDIC study. Ann Rheum Dis. 2016;75(10):1777-1782.

17. WHO. Recommendations on Treatment. Guidelines for the Screening Care and Treatment of Persons with Chronic Hepatitis C Infection. Updated version. Geneva: World Health Organization; 2014:63-79.

18. Cacoub P, Comarmond C, Domont F, Savey L, Saadoun D. Cryoglobulinemia vasculitis. Am J Med. 2015;128(9):950-955.

19. Fabrizi F, Martin P, Cacoub P, Messa P, Donato FM. Treatment of hepatitis C-related kidney disease. Expert Opin Pharmacother. 2015; 16(12):1815-1827.

20. Fabrizi F, Plaisier E, Saadoun D, Martin P, Messa P, Cacoub P. Hepatitis $\mathrm{C}$ virus infection, mixed cryoglobulinemia, and kidney disease. Am J Kidney Dis. 2013;61(4):623-637.

21. Sneller MC, Zonghui H, Langford CA, et al. A randomized controlled trial of rituximab following failure of antiviral therapy for hepatitis C-associated cryoglobulinemic vasculitis. Arthritis Rheum. 2012;64(3):835-842.

22. De Vita S, Quartuccio L, Isola M, et al. A randomized controlled trial of rituximab for the treatment of severe cryoglobulinemic vasculitis. Arthritis Rheum. 2012;64(3):843-853.

23. Sansono D, De Re V, Lauletta G, et al. Monoclonal antibody treatment of mixed cryglobulinemia resistant to interferon $\alpha$ with an anti-CD20. Blood. 2003;101:3818-3826. 
24. Saadoun D, Resche Rigon M, Thibault V, et al. Peg-IFN $\alpha /$ ribavirin/ protease inhibitor combination in hepatitis $\mathrm{C}$ virus associated mixed cryoglobulinemia vasculitis: results at week 24. Ann Rheum Dis. 2014;73(5):831-837.

25. Saadoun D, Resche Rigon M, Sene D, et al. Rituximab plus Peg-interferonalpha/ribavirin compared with Peg-interferon-alpha/ribavirin in hepatitis C-related mixed cryoglobulinemia. Blood. 2010;116(3):326-334.

26. Terrier B, Saadoun D, Sène D, et al. Efficacy and tolerability of rituximab with or without PEGylated interferon alfa-2b plus ribavirin in severe hepatitis C virus-related vasculitis: a long-term follow-up study of thirty-two patients. Arthritis Rheum. 2009;60(8):2531-2540.

27. Mazzaro C, Zorat F, Caizzi M, et al. Treatment with peg-interferon alfa$2 \mathrm{~b}$ and ribavirin of hepatitis $\mathrm{C}$ virus-associated mixed cryoglobulinemia: a pilot study. J Hepatol. 2005;42(5):632-638.

28. Dammacco F, Tucci FA, Lauletta G, et al. Pegylated interferon-alpha, ribavirin, and rituximab combined therapy of hepatitis $C$ virus-related mixed cryoglobulinemia: a long-term study. Blood. 2010;116(3):343-353.

29. Tallarita T, Gagliano M, Corona D, et al. Successful combination of rituximab and plasma exchange in the treatment of cryoglobulinemic vasculitis with skin ulcers: a case report. Cases J. 2009;2:7859.

30. Fortea-Ormaechea JI, Lozano-Maya M, Adán-Merino L, et al. Long-term remission with rituximab in a patient with severe hepatitis $\mathrm{C}$ virus-induced mixed cryoglobulinemia. Rev Esp Enferm Dig. 2013;105(8):490-493.

31. Bonilla-Abadía F, Echeverri AF, Izquierdo JH, Cañas F, Cañas CA. Efficacy and safety of rituximab in the treatment of vasculitic leg ulcers associated with hepatitis $\mathrm{C}$ virus infection. Case Rep Rheumatol. 2012;2012:923897.

32. Urraro T, Gragnani L, Piluso A, et al. Combined treatment with antiviral therapy and rituximab in patients with mixed cryoglobulinemia: review of the literature and report of a case using direct antiviral agents-based antihepatitis C virus therapy. Case Rep Immunol. 2015;2015:816424.

33. Fabrizi F, Fogazzi GB, Cresseri D, et al. Antiviral therapy for HCVassociated cryoglobulinemic glomerulonephritis: case report and review of the literature. Kidney Blood Press Res. 2012;35(6):687-693.

34. Lamprecht $P$, Lerin-Lozano $C$, Merz H, et al. Rituximab induces remission in refractory $\mathrm{HCV}$ associated cryoglobulinaemic vasculitis. Ann Rheum Dis. 2003;62(12):1230-1233.

35. Saadoun D, Pineton de Chambrun M, Hermine O, et al. Using rituximab plus fludarabine and cyclophosphamide as a treatment for refractory mixed cryoglobulinemia associated with lymphoma. Arthritis Care Res. 2013;65(4):643-647.

36. Cruz B, de Melo AL, de Almeida Cruz Filho A, et al. Non-hepatitis C virus crioglobulinemic vasculitis: a case series of nine patients. Rev Bras Reumatol. 2006;46(5):310-314.
37. Ferri C, Cacoub P, Mazzaro C, et al. Treatment with rituximab in patients with mixed cryoglobulinemia syndrome: results of multicenter cohort study and review of the literature. Autoimmun Rev. 2011;11(1): 48-55.

38. Terrier B, Krastinova E, Marie I, et al. Management of noninfectious mixed cryoglobulinemia vasculitis: data from 242 cases included in the CryoVas survey. Blood. 2012;119(25):5996-6004.

39. Koukoulaki M, Abeygunasekara SC, Smith KG, et al. Remission of refractory hepatitis C-negative cryoglobulinaemic vasculitis after rituximab and infliximab. Nephrol Dial Transplant. 2005;20(1): 213-216.

40. Pasquet F, Combarnous F, MacGregor B, et al. Safety and efficacy of rituximab treatment for vasculitis in hepatitis B virus-associated type II cryoglobulinemia: a case report. J Med Case Reports. 2012; 6:39.

41. Bitar ZI, Alajmi M, Maadarani O, et al. Rituximab-induced hepatitis C virus fulminant hepatitis (case report). JCRM. 2014;2014.

42. Terrier B, Launay D, Kaplanski G, et al. Safety and efficacy of rituximab in nonviral cryoglobulinemia vasculitis: data from the French Autoimmunity and Rituximab registry. Arthritis Care Res. 2010;62(12):1787-1795.

43. Fabrizi F, Cresseri D, Fogazzi GB, et al. Rituximab therapy for primary glomerulonephritis: report on two cases. World J Clin Cases. 2015;3(8):736-742.

44. Sène D, Ghillani-Dalbin P, Amoura Z, Musset L, Cacoub P. Rituximab may form a complex with IgMkappa mixed cryoglobulin and induce severe systemic reactions in patients with hepatitis $\mathrm{C}$ virus-induced vasculitis. Arthritis Rheum. 2009;60(12):3848-3855.

45. Ostojic P. Cryoglobulinemic vasculitis in systemic sclerosis successfully treated with mycophenolate mofetil. Rheumatol Int. 2014; 34(1):145-147.

46. Bartolucci P, Ramanoelina J, Cohen P, et al. Efficacy of the anti-TNF- $\alpha$ antibody infliximab against refractory systemic vasculitidis: an open pilot study on 10 patients. Rheumatology. 2002;41(10):1126-1132.

47. Chandesris MO, Gayet S, Schleinitz N, et al. Infliximab in the treatment of refractory vasculitis secondary to hepatitis C-associated mixed cryoglobulinaemia. Rheumatology. 2004;43(4):532-533.

48. Gragnani L, Piluso A, Giannini C, et al. Genetic determinants in hepatitis $\mathrm{C}$ virus-associated mixed cryoglobulinemia: role of polymorphic variants of BAFF promoter and Fc $\gamma$ receptors. Arthritis Rheum. 2011;63(5): 1446-1451.

49. Fabris M, Quartuccio L, Sacco S, et al. B-Lymphocyte stimulator (BLyS) up-regulation in mixed cryoglobulinaemia syndrome and hepatitis-C virus infection. Rheumatology (Oxford). 2007;46(1):37-43.
Journal of Inflammation Research

\section{Publish your work in this journal}

The Journal of Inflammation Research is an international, peer-reviewed open access journal that welcomes laboratory and clinical findings on the molecular basis, cell biology and pharmacology of inflammation including original research, reviews, symposium reports, hypothesis formation and commentaries on: acute/chronic inflammation; mediators of

\section{Dovepress}

inflammation; cellular processes; molecular mechanisms; pharmacology and novel anti-inflammatory drugs; clinical conditions involving inflammation. The manuscript management system is completely online and includes a very quick and fair peer-review system. Visit http://www.dove press.com/testimonials.php to read real quotes from published authors. 